1 Convergent inactivation of the skin-specific C-C motif chemokine ligand 27 in

\title{
mammalian evolution
}

3

4 Mónica Lopes-Marques ${ }^{1+*}$, Luís Q. Alves ${ }^{1,2+}$, Miguel M. Fonseca ${ }^{1}$, Giulia Secci-

5 Petretto $^{1,2}$, André M. Machado ${ }^{1,2}$, Raquel Ruivo ${ }^{1 *}$ and L. Filipe C. Castro ${ }^{1,2^{*}}$

6

$7{ }^{1}$ CIIMAR - Interdisciplinary Centre of Marine and Environmental Research, U. Porto -

8 University of Porto, Porto, Portugal

$9 \quad{ }^{2}$ Department of Biology, Faculty of Sciences, U. Porto - University of Porto, Portugal

10

$11{ }^{+}$These authors contributed equally to this work

12

$13{ }^{*}$ To whom correspondence should be addressed:

14 L. Filipe C. Castro, Raquel Ruivo and Mónica Lopes-Marques

15 CIIMAR, Av. General Norton de Matos s/n; 4450-208, Matosinhos, Portugal

16 phone (+351) 223401800

17 Email: filipe.castro@ciimar.up.pt; ruivo.raquel@gmail.com; monicaslm@hotmail.com

19 Running title: CCL27 gene loss in mammals

20 Keywords: chemokines, gene loss, skin, inflammation

21 


\section{Abstract}

24 The appearance of mammalian-specific skin features was a key evolutionary event

25 contributing for the elaboration of physiological processes such as thermoregulation,

26 adequate hydration, locomotion and inflammation. Skin inflammatory and autoimmune

27 processes engage a population of skin-infiltrating $\mathrm{T}$ cells expressing a specific $\mathrm{C}-\mathrm{C}$

28 chemokine receptor (CCR10), which interacts with an epidermal CC chemokine, the skin-

29 specific C-C motif chemokine ligand 27 (CCL27). CCL27 is selectively produced in the

30 skin by keratinocytes, particularly upon inflammation, mediating the adhesion and

31 homing of skin-infiltrating T cells. Here, we examined the evolution and coding condition

32 of $C c l 27$ in 112 placental mammalian species. Our findings reveal that a number of open

33 reading frame inactivation events such as insertions, deletions, start and stop codon

34 mutations, independently occurred in Cetacea, Pholidota, Sirenia, Chiroptera, and

35 Rodentia, totalizing 18 species. The diverse habitat settings and life-styles of Ccl27-

36 eroded lineages probably implied distinct evolutionary triggers rendering this gene

37 unessential. For example, in Cetacea the rapid renewal of skin layers minimizes the need

38 for an elaborate inflammatory mechanism, mirrored by the absence of epidermal scabs.

39 Our findings suggest that the convergent and independent loss of $\mathrm{Ccl} 27$ in mammalian

40 evolution concurred with unique adaptive roads for skin physiology. 


\section{Introduction}

42 The mammalian skin performs a plethora of biological functions, including that of acting as a protective barrier from external harmful insults, such as invading pathogens and noxious stimuli. In this context, the role of the immune system is fundamental, involving a coherent and highly coordinated network of innate and adaptive components to ensure an adequate response to ensure homeostasis [1, 2]. Chemokines, a superfamily of polypeptides, are central in the unfolding of immune and inflammatory responses, serving as chemoattractant signals that drive the movements of immune cells in response to stimuli. In the skin, a tissue-specific $\mathrm{T}$ cell homing chemokine has been described. Initially named as the cutaneous $\mathrm{T}$ cell-attracting chemokine, $\mathrm{C}-\mathrm{C}$ motif chemokine 51 ligand 27 (CCL27, also known as ESkine, ALP, ILC or ILR $\alpha$ locus chemokine [3-5]) plays a central role in the skin homing process [6]. Ccl27 maps to human chromosome 9 in a tandem gene arrangement with two other chemokines, $C c l 19$ and $C c l 21$ and presents two alternative transcripts, yielding secreted and intracellular forms (Figure 1) [3, 7]. The latter, designated PESKY, includes a different exon 1 and acts as an intracellular chemokine (Figure 1) [7]. PESKY transcripts may be found in various mucosal tissues $[8,9]$, but CCL27 secretion is mostly restricted to skin keratinocytes, having a critical role in skin homeostasis $[6,10]$. To provide a skin-specific cue to attract memory $\mathrm{T}$ cells in normal or inflamed skin, CCL27 specifically binds to the CCR10 receptor in vivo [6, 10]. While CCL27 is exclusive towards CCR10 receptor, other chemokines such as CCL8 also bind CCR10 $[6,9,10]$.

62 While a number of key morpho-functional skin components have been conserved

63 throughout mammalian evolution, specific lineages experienced secondary episodes of

64 phenotypic simplification or/and elaboration (e.g. [11, 12]). In this context, Cetacea offer 65 an illustrative example, with the exclusive aquatic dependence underscoring unique 
anatomical signatures (e.g. $[12,13])$. For example, their skin is smooth with no pelage,

67 presenting a thick stratum corneum, while the upper layers of the epidermis are not fully cornified [14-16]. Moreover, to improve smoothness and reduce drag, Cetacea skin is rapidly renewed $[15,17,18]$. This intensive cellular replacement and epidermal thickness reduce scab formation and the risk of pathogen invasion $[14,16,18]$. Accordingly, skin inflammation is apparently reduced in Cetacea [18]. The underlying genomic events connected with skin repair mechanisms and whether other mammalian lineages display similar traits is presently unknown. The growing number of full genome sequences currently available have provided valuable insights into the role of gene loss as the foundation for phenotypic alterations and consequently on the perception of adaptive landscapes $[11,12,19-22]$. Given the key role of CCL27 in the process of skin inflammation, we hypothesized that the $C c l 27$ coding sequence might be compromised in Cetacea as suggested by the overall skin inflammatory physiology observed in this lineage $[15,18]$.

\section{Results and Discussion}

82 To investigate the distribution and annotation tags of the $C c l 27$ gene in mammals, we scrutinized a total of 114 selected mammalian genomes available at NCBI and Ensembl genome browsers (supplementary table 1). This search retrieved 14 Ccl27 annotations tagged as "low-quality" (LQ) and uncovered 9 species with no $C c l 27$ gene annotation

86 (supplementary table 1). Next, we investigated the genomic sequences corresponding to the $C c l 27$ LQ annotations to determine the CDS through manual annotation. This step revealed coding Ccl27 genes, tagged as LQ for the following species: Saimiri boliviensis

89 (black-capped squirrel monkey), Galeopterus variegatus (Sunda flying lemur),

90 Peromyscus maniculatus bairdii (North American deer mouse), Loxodonta Africana 
91 (African bush elephant), and Chrysochloris asiatica (Cape golden mole). Also, the

92 analysis of the genomic sequence corresponding to the Ccl27 locus in Ochotona princeps

93 (American pika) showed that the missing annotation in this species is most probably due

94 to poor genome coverage in this locus (not shown). Importantly, all cetacean species

95 analysed presented sequences tagged as LQ or no Ccl27 annotation. This impelled us to

96 further explore other cetacean species with unannotated genomes: Balaenoptera

97 bonaerensis (Antarctic minke whale), Eschrichtius robustus (gray whale), Balaena mysticetus (bowhead whale) and Sousa chinensis (Indo-Pacific humpback dolphin).

$100 \mathrm{Ccl} 27$ gene sequence contains inactivating mutations in Cetacea

101 Annotation of collected cetacean genomic sequences revealed $C c l 27$ gene erosion across 102 all analysed species (Figure 2A). In detail, gene sequence examination in Odontoceti 103 showed a non-disruptive insertion of a codon in exon 2 in all species with the exception 104 of Lipotes vexillifer (Yangtze river dolphin). In addition, in exon 2, a frameshift mutation 105 (deletion of 1 nucleotide) was identified and validated by sequence read archive (SRA) 106 analysis in Physeter catodon (sperm whale, supplementary material 1). A conserved 107 premature stop codon was found in Orcinus orca (orca) and Lagenorhynchus obliquidens 108 (Pacific white-sided dolphin), as well as a non-conserved premature stop codon in $L$. 109 vexillifer. These observations were confirmed in O. orca and L. obliquidens through SRA 110 analysis (supplementary material 1). In L. vexillifer exon 2 also presented a frameshift 111 mutation (4 nucleotide deletion) and the loss of the canonical splice site (GT $>C C)$. Next, 112 in exon 3 a conserved premature stop codon was identified in all Odontoceti (Figure 2B,

113 supplementary material 1). A frameshift mutation by deletion was identified in all species 114 apart from $P$. catodon, which in turn shows a frameshift mutation before the identified 115 stop codon (Figure 2B). 
116 Regarding the Mysticeti, all identified mutations were conserved across all 4 analyzed

117 species (Figure 2A and 2C). Non-disruptive mutations consisting in the deletion and

118 insertion of 1 codon were identified in exon 1 and exon 2, respectively. Also, two

119 conserved premature stop codons were identified in exon 2 and exon 3 (the former was

120 validated by SRA, supplementary material 1), which were followed by a 1 nucleotide

121 deletion identified in all analyzed species (Figure 2C black arrow). Interestingly, this 1

122 nucleotide deletion is conserved among all cetacean species (supplementary material 2),

123 suggesting that $C c l 27$ pseudogenization preceded the divergence of Odonticeti and

124 Mysticeti.

126 Transcriptomic analysis supports $\boldsymbol{C c l} 27$ gene erosion in Cetacea

127 To further scrutinize the functional condition of $C c l 27$, we next analyzed multi-tissue

128 RNA-Seq projects available at NCBI for 6 cetacean species: Tursiops truncatus (common

129 bottlenose dolphin), Delphinapterus leucas (beluga whale), Neophocaena asiaeorientalis

130 (finless porpoise), P. catodon (sperm whale), Balaenoptera acutorostrata (common

131 minke whale), and B. mysticetus (supplementary table 2).

132 Overall, RNA-Seq analysis revealed a considerably low number of $C c l 27$ mRNA reads

133 across all the 6 species, especially in N. asiaeorientalis (Figure 3). Moreover, for the

134 remaining species we observed a substantially high proportion of reads spanning adjacent

135 exonic and intronic regions, exon-intron reads, versus spliced reads, connecting

136 contiguous exons and containing no intronic remnants, especially in the case of $T$.

137 truncatus (121 exon-intron reads against 3 spliced reads). In the later, the higher number

138 of skin-specific sequencing runs available for this species, compared to the remaining

139 ones (25 skin-specific sequencing runs in T. truncatus vs. an average of 6.6 skin-specific

140 sequencing runs per species), probably explains the variation in the number of exon- 
141 intron reads (see supplementary table 2). As we observed a specific case with a

142 considerably distinct ratio of exon-intron reads/spliced reads amongst the remaining

143 species, namely B. mysticetus (49 spliced reads vs 52 exon-intron reads), we decided to

144 further verify the presence of ORF disruptive mutations in the produced transcripts of

145 Ccl27 in each of the referred species. We were able to detect at least one premature stop

146 codon in the transcripts of the analysed 6 cetacean species (see supplementary material

147 3), revealing that Ccl27 transcripts contained the genome predicted ORF mutations

148 (Figure 3).

149 The conserved mutational pattern observed between Odontoceti and Mysticeti suggests

150 that $\mathrm{Ccl} 27$ inactivation occurred in the Cetacea ancestor. To further survey and estimate

151 the approximate timing of $C c l 27$ loss in Cetacea, we next investigated the genome and

152 the skin transcriptome of the extant sister clade of the Cetacea, the Hippopotamidae. The

153 current version of the H. amphibius genome, available at NCBI, is fragmented and

154 unannotated (GCA_002995585.1). However, we were able to deduce the full coding ORF

155 of the $C c l 27$ gene orthologue in H. amphibius, and without any intervening inactivating

156 mutation (Figure 3). Furthermore, by examining a skin-specific transcriptome we

157 identified a very high proportion of spliced/exon-intron mRNA reads (1995 spliced reads

158 against 379 exon-intron reads), a clear indication that the gene is functional in this species

159 (Figure 3).

160

$161 \mathrm{Ccl} 27$ is eroded in other non-cetacean mammals

162 We next investigated the uniqueness of $\operatorname{Ccl} 27$ inactivation in other mammalian lineages

163 with absent or LQ annotations. Our initial analysis revealed that several genes annotated

164 as LQ were in fact coding. For example, the analysis of the retrieved genomic sequence

165 for L. africana revealed poor genome coverage in the Ccl27 locus. However, blast search 
166 of the whole genome sequence recovered a genomic scaffold

167 (NW_003573426.1:65683000-65687000) that contained an intact Ccl27 gene sequence.

168 Yet, in the case of LQ tagged Ccl27 from Hipposideros armiger (great groundleaf bat),

169 Trichechus manatus (West Indian manatee), Heterocephalus glaber (naked mole rat), and

170 Manis javanica (Sunda pangolin) the analysis and manual annotation of the

171 corresponding genomic sequence revealed a number of ORF disrupting mutations (Figure

172 4A). Our findings were further supported by searching the available unannotated genomes

173 of Manis pentadactyla (Chinese pangolin) and Rhinolophus sinicus (Chinese rufous

174 horseshoe bat), which after annotation also presented a non-coding Ccl27 ORF (Figure

175 4A). Briefly, Ccl27 annotation in H. armiger revealed a premature stop codon in exon 3

176 followed by 1 nucleotide insertion, and in $R$. sinicus a single premature stop codon was

177 identified in exon 2 (all confirmed by SRA search Supplementary material 4). In the

178 Pholidota M. javanica and M. pentadactyla a shared frameshift mutation in exon 2 was

179 identified (validated by SRA in M. javanica Supplementary material 4). Additionally, $M$.

180 pentadactyla presents a premature stop codon in exon 2 while $M$. javanica presents a

181 premature stop codon in exon 3 preceded by a 1 nucleotide frameshift mutation. In

182 rodentia, the $C c l 27$ gene annotation in $H$. glaber revealed a missing start codon in exon 1

183 combined with a premature stop codon in exon2, and finally in T. manatus Ccl27 gene

184 annotation uncovered a premature stop codon in exon 3 (stop codons validated by SRA

185 Supplementary material 4).

186

187 Exon 3 length reduction parallels gene inactivation

188 The survey of 112 placental mammalian Ccl27 CDS exposed a variable C-terminal length

189 in different mammalian species. Thus, we compared the predicted length of exon 3 in the

190 annotated pseudogenes regardless of prior ORF disrupting mutations (Figure 4B). This 
analysis showed that all annotated pseudogenes were severely truncated in exon 3 with the exception of $R$. sinicus. Also, the analysis of the observed truncations in the overall

193 structure of CCL27 using homology modelling for O. orca and B. mysticetus showed that

194 premature stop codons occur early in the C-terminal $\alpha$-helix. CC chemokines present a

195 highly conserved quaternary structure characterized by disordered N-terminal region

196 followed by a 310 -helix, 3 antiparallel $\beta$-strands, followed a C-terminal $\alpha$-helix and ending

197 with a disordered stretch of positive residues [23]. Interestingly, the C-terminal region,

198 specifically the disordered region, is a feature that differentiates CCL27 from the majority

199 of CC chemokines, and has been shown to be involved in nuclear import [9]. In

200 agreement, both Ccl27 transcript variants, the intracellular chemokine PESKY and the

201 internalized CCR10-bound CCL27, target the cell nucleus, modulating morphology and

202 motility via transcriptional modification [4, 8]. Moreover, the remaining mammals

203 including Tenricidae, Antilopinae, Caprinae, Platyrrhini, exhibit a sequence deletion

204 pattern at the end or shortly after the $\alpha$-helix (Figure 4B), which implies the loss of the

205 final C-terminal disordered region involved in nuclear targeting. Yet, contrarily to the

206 annotated pseudogenes, the coding Ccl27 Aotus nancymaae, which presents the shortest

207 exon 3 , still conserves the full $\alpha$-helix which has been reported to stabilize the overall fold

208 [23] (Figure 4C). The biological significance of this plasticity remains to be studied.

$210 \mathrm{Ccl} 27$ gene loss correlates with alternative protection and healing programs

211 Together, our analysis indicates that $\mathrm{Ccl} 27$ is most likely non-functional in all of the

212 examined cetacean species. Inactivating mutations are also present in species of

213 Pholidota, Sirenia, Chiroptera, and Rodentia. Even if a full phenotypic description of

214 mouse knock-out (KO) for this gene is presently unavailable, the initial data suggests a

215 decrease of the $\mathrm{T}$ cell population in intact skin [24]. On the other hand, constitutive 
216 production of keratinocyte CCL27 enhanced the inflammatory response in mice [25]. In

217 agreement, chronic inflammatory skin diseases, such as atopic dermatitis and psoriasis,

218 are characterized by increased serum levels of the T cell attracting chemokine CCL27

219 [10], while CCL27-neutralizing antibody treatment reduced skin inflammation in a 220 transgenic animal model [26]. Thus, upon insult or infection, Ccl27 KO would likely

221 show an attenuated inflammatory response in the skin. This hypothesis remains to be verified.

223 Nevertheless, it could be argued that the premature stop codon in exon 3 of $C c l 27$ in $T$.

224 truncatus, D. leucas, S. chinensis and N. asiaorientalis could still encode a functional

225 shorter isoform. Yet, RNA-Seq transcriptome and structural analysis supports a different

226 interpretation. Since $\mathrm{Ccl} 27$ prime expression site is the skin, we analysed the available

227 skin and multi-tissue RNA-transcriptomes from Cetacea and found two distinct scenarios.

228 First, in the majority of the species, RNA-Seq searches recovered reads covering exon-

229 intron. Second, in B. mysticetus the recovered RNA-Seq reads presented a higher number

230 of spliced reads. However, in both cases, detailed analysis of the collected reads

231 confirmed the presence of the previously identified ORF-disrupting mutations. Thus, we

232 suggest that these mRNA mature sequences do not translate into a functional protein. In

233 addition, previous studies, addressing the loss of visual opsins in Chiroptera, highlighted

234 possible discrepancies between gene integrity and protein production: further suggesting

235 post-transcriptional mechanisms as regulators of evolutionary gene silencing [22].

236 Our analysis strongly supports that Ccl27 gene pseudogenization compromises both 237 canonical CCL27 and PESKY transcripts. This is in accordance with previous findings 238 reporting a distinct inflammatory and wound healing program in cetacean skin $[18,27]$.

239 Interestingly, scar-less and low inflammation wound repair has also been reported in 240 several mammalian foetus, including human, as well as in human adult oral mucosa [28, 
241 29]. Both observations might correlate with decreased or null CCL27 secretion. In fact,

242 embryonic keratinocytes are more proliferative and less immunogenic than adult cells,

243 inhibiting $\mathrm{T}$ cell proliferation [30]. Similarly, oral mucosa exhibits rapid wound healing

244 due to accelerated re-epithelialization [28]. In wounded oral mucosa, the overall

245 expression of $\mathrm{Ccl} 27$ is also downregulated when compared to wounded skin [28]. Thus,

246 in scab-less and low inflammation wound repair, increased epithelial renewal seems to

247 parallel the downregulation or absence of CCL27 secretion. Yet, in oral mucosa the

248 possible maintenance of PESKY could participate in the healing circuitry by stimulating

249 cell migration and proliferation.

250 Additionally, we found convergent inactivation of $\mathrm{Ccl} 27$ in other non-cetacean

251 mammalian species: namely in pangolins (M. javanica and M. pentadactyla), in the naked

252 mole rat (H. glaber), in the sirenian T. manatus, and in two Chiroptera (H. armiger and

253 R. sinicus). Curiously, with the exception of Chiroptera, these species share some of the

254 distinctive features of Cetacea skin: for example, the hairless phenotype is observed in

255 Pholidota, Sirenia, and naked mole rat, increased epidermal thickness is observed in

256 Sirenia and naked mole rat and Sirenia skin is also smooth [14, 31]. The diversity of skin

257 phenotypes along with the scarce information regarding species-specific inflammatory

258 and wound healing programs, hampers the anticipation of the possible outcomes of Ccl27

259 pseudogenization. Nonetheless, the available information suggests that $C c l 27$ erosion

260 occurred in species exhibiting singular epidermal renewal, or even protective mechanisms

261 or structures, reducing the need for CCL27-dependent inflammatory processes. For

262 instance, pangolins present a protective armour with keratin-derived scales, which was

263 suggested to reduce epithelial immune requirements [32, 33]. In agreement,

264 pseudogenization of Interferon Epsilon, which confers protection against viral and

265 bacterial infections, was also reported in these species [33]. On the other hand, the naked 
mole rat abundantly produces high molecular weight hyaluronic acid, suggested to underscore their peculiar longevity and cancer resistance but also contributing to cell motility, rapid wound healing and immunity [31, 34]. Regarding Chiroptera, although

269 their skin is generally similar to most mammalian species, interdigital skin membranes 270 are thinner, and thus more susceptible to damage; yet, interdigital membranes have an 271 enhanced healing capacity [35]. Nonetheless, the inflammatory circuitry of this healing process is still poorly studied. Also, Ccl27 pseudogenization was only detected in two

273 Chiroptera species. Again, post-translational events could promote CCL27 loss in 274 additional species [22]. In conclusion, our findings reinforce gene loss mechanisms as 275 evolutionary drivers of skin phenotypes in mammals, and correlate $C c l 27$ loss with 276 species-specific scar-less and/or low inflammation wound repair.

\section{Material and Methods}

\section{Sequence retrieval}

280 Ccl27 coding nucleotide sequences were searched and collected from NCBI for a set of 281 mammalian species representative of the major mammalian lineages (see Supplementary table 1). Searches were performed through tblastn and blastn queries using the human

283 Ccl27 sequence as reference. Full coding sequences and corresponding genomic 284 sequences were collected, for phylogenetic analysis and gene annotation respectively. 285 Coding sequences were next uploaded into Geneious R7.1.9 curated by removing 5'and 286 3' UTR (untranslated regions) and aligned using the translation align option. Sequence 287 alignment was inspected and exported for phylogenetic analysis. Maximum likelihood 288 Phylogenetic analysis was performed in PhyML3.0 server [36], with best sequence 289 evolutionary model determined using smart model selection [37], and branch support with 
290 the aBayes algorithm [38]. The resulting phylogenetic tree was then visualized and

291 analysed in Figtree (Supplementary material 5).

\section{Gene annotation}

293 For gene annotation the genomic sequence of $C c l 27$ annotations tagged as LQ was

294 collected from NCBI. For species with no Ccl27 annotation (B. acutorostrata, O. orca

295 and $R$. sinicus), the genomic sequence ranging from the upstream to the downstream

296 flanking genes was collected. Finally, for species with no annotated genome (B.

297 bonaerensis, E. robustus, B. mysticetus, H. amphibius and M. pentadactyla), genomic

298 sequences were recovered through tblastn searches in the whole genome assembly and

299 scaffold corresponding to the highest identity hits were taken. Collected genomic

300 sequences were next loaded to Geneious R7.1.9 for manual annotation as previously

301 described [11, 39]. Briefly, using as reference human and Bos taurus Ccl27 CDS

302 sequence as reference each individualized exon was mapped on the corresponding

303 genomic sequences using the built-in map to reference tool in Geneious R7.1.9. Aligned

304 regions were manually inspected to verify coding status and identify ORF disrupting

305 mutations (frameshifts, premature stop codon, loss of canonical splice sites). The

306 identified mutations were next validated in at least two independent SRA projects (when

307 available) (see supplementary material 1).

308 Transcriptomic Analysis

309 RNA-Seq analysis was performed to assess the functional condition of $\mathrm{Ccl} 27$ in 6

310 cetacean species and H. amphibius. For each of the 6 cetaceans, using the discontiguous

311 megablast task from Blastn, the B. taurus Ccl27 coding sequence (CDS) was used as

312 query sequence to recover reads from the totality of the available transcriptomic sequence

313 read archive (SRA) projects available at NCBI. The supplementary table 2 provides an

314 in-depth description of the explored NCBI SRA projects per species. In the case of $H$. 
315 amphibius, through megablast from Blastn, the CDS of the annotated gene in the same

316 species was used as query sequence and reads were recovered from the available $H$.

317 amphibius skin transcriptome (accession number PRJNA507170). The collected mRNA

318 reads were mapped against the corresponding annotated gene using the map to reference

319 tool from Geneious R7.1.9. The aligned regions were manually curated, and poorly

320 aligning reads manually removed. Next reads were then classified as spliced reads (reads

321 spanning over two different exons) and exon-intron reads (reads containing intronic

322 sequence). Reads fully overlapping a single exon, exonic reads, were considered

323 inconclusive for this analysis, given that it is infeasible to infer the nature of the

324 corresponding transcript (spliced or unspliced).

\section{Comparative homology modelling}

326 Comparative homology modelling was performed for $O$. orca representative of

327 Odontoceti, B. mysticetus representative of Mysticeti and for Aotus nancymaae

328 (Platyrhini) representing a coding CDS with short C-terminal. Predicted CDS sequences

329 of $O$. orca and B. mysticetus were determined using the annotated exons and premature

330 stop codons identified in exon 2 were reverted to the residue observed in B. taurus, while

331 mutations in exon 3 were left as observed. Corresponding protein sequences were then

332 next submitted to the SWISS-MODEL [40, 41] for homology modelling using the human

333 CCL27 crystal structure as reference (2KUM) [23]. Resulting models were downloaded

334 and analysed in PyMOL V1.74 [42].

336 Acknowledgments

337 This work was supported by Project No. 031342 co-financed by COMPETE 2020,

338 Portugal 2020 and the European Union through the ERDF, and by FCT through national 339 funds. 
342 The authors declare no competing interests.

References

344

1. Di Meglio P, Perera GK, Nestle FO: The multitasking organ: recent insights into skin immune function. Immunity 2011, 35:857-869.

2. Pasparakis M, Haase I, Nestle FO: Mechanisms regulating skin immunity and inflammation. Nat Rev Immunol 2014, 14:289-301.

3. Baird JW, Nibbs RJB, Komai-Koma M, Connolly JA, Ottersbach K, ClarkLewis I, Liew FY, Graham GJ: ESkine, a Novel $\beta$-Chemokine, Is Differentially Spliced to Produce Secretable and Nuclear Targeted Isoforms. Journal of Biological Chemistry 1999, 274:33496-33503.

4. Hromas R, Broxmeyer HE, Kim C, Christopherson K, 2nd, Hou YH: Isolation of ALP, a novel divergent murine $C C$ chemokine with a unique carboxy terminal extension. Biochem Biophys Res Commun 1999, 258:737-740.

5. Ishikawa-Mochizuki I, Kitaura M, Baba M, Nakayama T, Izawa D, Imai T, Yamada H, Hieshima K, Suzuki R, Nomiyama H, Yoshie O: Molecular cloning of a novel CC chemokine, interleukin-11 receptor alpha-locus chemokine (ILC), which is located on chromosome 9p13 and a potential homologue of a CC chemokine encoded by molluscum contagiosum virus. FEBS Lett 1999, 460:544-548.

6. Morales J, Homey B, Vicari AP, Hudak S, Oldham E, Hedrick J, Orozco R, Copeland NG, Jenkins NA, McEvoy LM, Zlotnik A: CTACK, a skinassociated chemokine that preferentially attracts skin-homing memory $T$ cells. Proceedings of the National Academy of Sciences 1999, 96:14470-14475.

7. Gortz A, Nibbs RJB, McLean P, Jarmin D, Lambie W, Baird JW, Graham GJ: The Chemokine ESkine/CCL27 Displays Novel Modes of Intracrine and Paracrine Function. The Journal of Immunology 2002, 169:1387-1394.

8. Ledee DR, Chen J, Tonelli LH, Takase H, Gery I, Zelenka PS: Differential expression of splice variants of chemokine CCL27 mRNA in lens, cornea, and retina of the normal mouse eye. Mol Vis 2004, 10:663-667.

9. Nibbs RJ, Graham GJ: CCL27/PESKY: a novel paradigm for chemokine function. Expert Opin Biol Ther 2003, 3:15-22.

10. Homey B, Alenius H, Muller A, Soto H, Bowman EP, Yuan W, McEvoy L, Lauerma AI, Assmann T, Bunemann E, et al: CCL27-CCR10 interactions regulate $\mathbf{T}$ cell-mediated skin inflammation. Nat Med 2002, 8:157-165.

11. Lopes-Marques M, Machado AM, Barbosa S, Fonseca MM, Ruivo R, Castro LFC: Cetacea are natural knockouts for IL20. Immunogenetics 2018.

12. Sharma V, Hecker N, Roscito JG, Foerster L, Langer BE, Hiller M: A genomics approach reveals insights into the importance of gene losses for mammalian adaptations. Nature Communications 2018, 9:1215.

13. McGowen MR, Gatesy J, Wildman DE: Molecular evolution tracks macroevolutionary transitions in Cetacea. Trends in Ecology \& Evolution 2014, 29:336-346.

14. Sokolov VE: Comparative morphology of skin of different orders: Ordo Cetacea. In Mammal Skin. Berkeley University of California Press Ltd.; 1982: 284-324 
15. Mouton M, Botha A: Cutaneous Lesions in Cetaceans: An Indicator of Ecosystem Status?; 2012.

16. Spearman RI: The epidermal stratum corneum of the whale. J Anat 1972, 113:373-381.

17. Hicks BD, St Aubin DJ, Geraci JR, Brown WR: Epidermal growth in the bottlenose dolphin, Tursiops truncatus. J Invest Dermatol 1985, 85:60-63.

18. Zabka TS, Romano TA: Distribution of MHC II (+) cells in skin of the Atlantic bottlenose dolphin (Tursiops truncatus): An initial investigation of dolphin dendritic cells. The Anatomical Record Part A: Discoveries in Molecular, Cellular, and Evolutionary Biology 2003, 273A:636-647.

19. Albalat R, Canestro C: Evolution by gene loss. Nat Rev Genet 2016, 17:379391.

20. Strasser B, Mlitz V, Fischer H, Tschachler E, Eckhart L: Comparative genomics reveals conservation of filaggrin and loss of caspase-14 in dolphins. Exp Dermatol 2015, 24:365-369.

21. Lachner J, Mlitz V, Tschachler E, Eckhart L: Epidermal cornification is preceded by the expression of a keratinocyte-specific set of pyroptosisrelated genes. Scientific Reports 2017, 7:17446.

22. Sadier A, Davies KTJ, Yohe LR, Yun K, Donat P, Hedrick BP, Dumont ER, Dávalos LM, Rossiter SJ, Sears KE: Multifactorial processes underlie parallel opsin loss in neotropical bats. eLife 2018, 7:e37412.

23. Jansma AL, Kirkpatrick JP, Hsu AR, Handel TM, Nietlispach D: NMR analysis of the structure, dynamics, and unique oligomerization properties of the chemokine CCL27. J Biol Chem 2010, 285:14424-14437.

24. Davila ML, Fu Y, Yang J, Xiong N: Role of CCR10 and CCL27 in skin resident $\mathbf{T}$ cell development and homeostasis. The Journal of Immunology 2016, 196:137.137-137.137.

25. Kagami S, Saeki H, Tsunemi Y, Nakamura K, Kuwano Y, Komine M, Nakayama T, Yoshie O, Tamaki K: CCL27-transgenic mice show enhanced contact hypersensitivity to Th2, but not Th1 stimuli. Eur J Immunol 2008, 38:647-657.

26. Chen L, Lin SX, Agha-Majzoub R, Overbergh L, Mathieu C, Chan LS: CCL27 is a critical factor for the development of atopic dermatitis in the keratin-14 IL-4 transgenic mouse model. Int Immunol 2006, 18:1233-1242.

27. Zasloff M: Observations on the Remarkable (and Mysterious) WoundHealing Process of the Bottlenose Dolphin. Journal of Investigative Dermatology 2011, 131:2503-2505.

28. Iglesias-Bartolome R, Uchiyama A, Molinolo AA, Abusleme L, Brooks SR, Callejas-Valera JL, Edwards D, Doci C, Asselin-Labat M-L, Onaitis MW, et al: Transcriptional signature primes human oral mucosa for rapid wound healing. Science Translational Medicine 2018, 10.

29. Moore AL, Marshall CD, Barnes LA, Murphy MP, Ransom RC, Longaker MT: Scarless wound healing: Transitioning from fetal research to regenerative healing. Wiley Interdiscip Rev Dev Biol 2018, 7.

30. Tan Kenneth KB, Salgado G, Connolly John E, Chan Jerry KY, Lane EB: Characterization of Fetal Keratinocytes, Showing Enhanced Stem Cell-Like Properties: A Potential Source of Cells for Skin Reconstruction. Stem Cell Reports 2014, 3:324-338. 
31. Daly TJ, Buffenstein R: Skin morphology and its role in thermoregulation in mole-rats, Heterocephalus glaber and Cryptomys hottentotus. J Anat 1998, 193 ( Pt 4):495-502.

32. Meyer W, Liumsiricharoen M, Suprasert A, Fleischer LG, Hewicker-Trautwein M: Immunohistochemical demonstration of keratins in the epidermal layers of the Malayan pangolin (Manis javanica), with remarks on the evolution of the integumental scale armour. European journal of histochemistry : EJH 2013, 57:e27-e27.

33. Choo SW, Rayko M, Tan TK, Hari R, Komissarov A, Wee WY, Yurchenko AA, Kliver S, Tamazian G, Antunes A, et al: Pangolin genomes and the evolution of mammalian scales and immunity. Genome research 2016, 26:1312-1322.

34. Fisher GJ: Cancer resistance, high molecular weight hyaluronic acid, and longevity. Journal of cell communication and signaling 2015, 9:91-92.

35. Ceballos-Vasquez A, Caldwell JR, Faure PA: Seasonal and reproductive effects on wound healing in the flight membranes of captive big brown bats. Biology Open 2015, 4:95.

36. Guindon S, Dufayard JF, Lefort V, Anisimova M, Hordijk W, Gascuel O: New Algorithms and Methods to Estimate Maximum-Likelihood Phylogenies: Assessing the Performance of PhyML 3.0. Systematic Biology 2010, 59:30721.

37. Vincent Lefort, Jean-Emmanuel Longueville, Olivier Gascuel: SMS: Smart Model Selection in PhyML. Molecular Biology and Evolution 2017, 34:24222424.

38. Anisimova M, Gil M, Dufayard JF, Dessimoz C, Gascuel O: aBayes: Survey of branch support methods demonstrates accuracy, power, and robustness of fast likelihood-based approximation schemes. Systematic Biology 2011, 60:685-99.

39. Lopes-Marques M, Ruivo R, Fonseca E, Teixeira A, Castro LFC: Unusual loss of chymosin in mammalian lineages parallels neo-natal immune transfer strategies. Mol Phylogenet Evol 2017, 116:78-86.

40. Waterhouse A, Bertoni M, Bienert S, Studer G, Tauriello G, Gumienny R, Heer FT, de Beer TAP, Rempfer C, Bordoli L, et al: SWISS-MODEL: homology modelling of protein structures and complexes. Nucleic Acids Res 2018, 46:W296-w303.

41. Benkert P, Biasini M, Schwede T: Toward the estimation of the absolute quality of individual protein structure models. Bioinformatics 2011, 27:343350 .

42. Schrodinger L: The PyMOL Molecular Graphics System. 2010. 


\section{$483 \quad$ Figures}

484

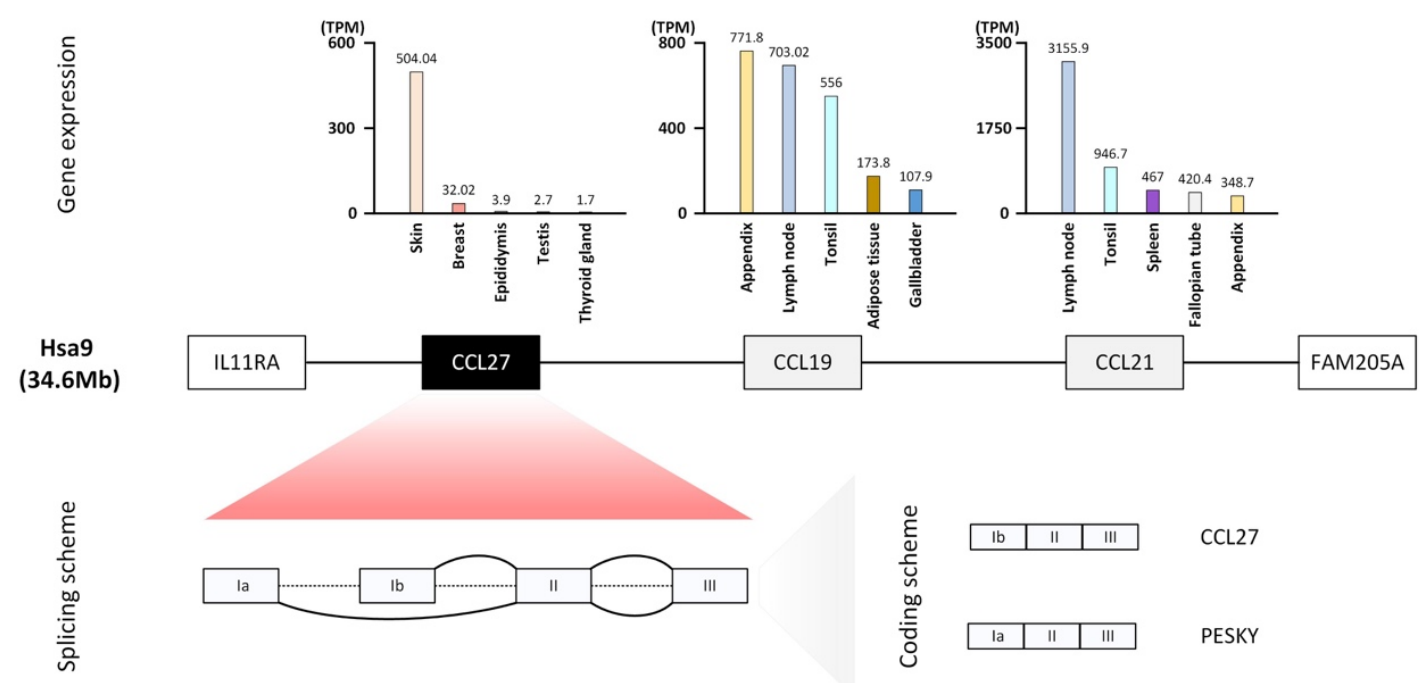

486 Figure 1: The human orthologue Ccl27 gene expression, genomic locus and structure. In 487 the centre, the genomic region of Homo sapiens at chromosome 9, containing Ccl27 gene 488 (in black box) and tandem gene duplicates, Ccl19 and Ccl21 (grey boxes). The 489 corresponding $\mathrm{Ccl}$ gene expression data was retrieved directly from the Human Protein 490 Atlas (https://www.proteinatlas.org/). Only five tissues with the highest values of 491 transcripts per million (TPM) are represented. Bottom figure represents $C c l 27$ gene 492 structure and alternative splicing producing two transcripts: CCL27 and PESKY. 493 

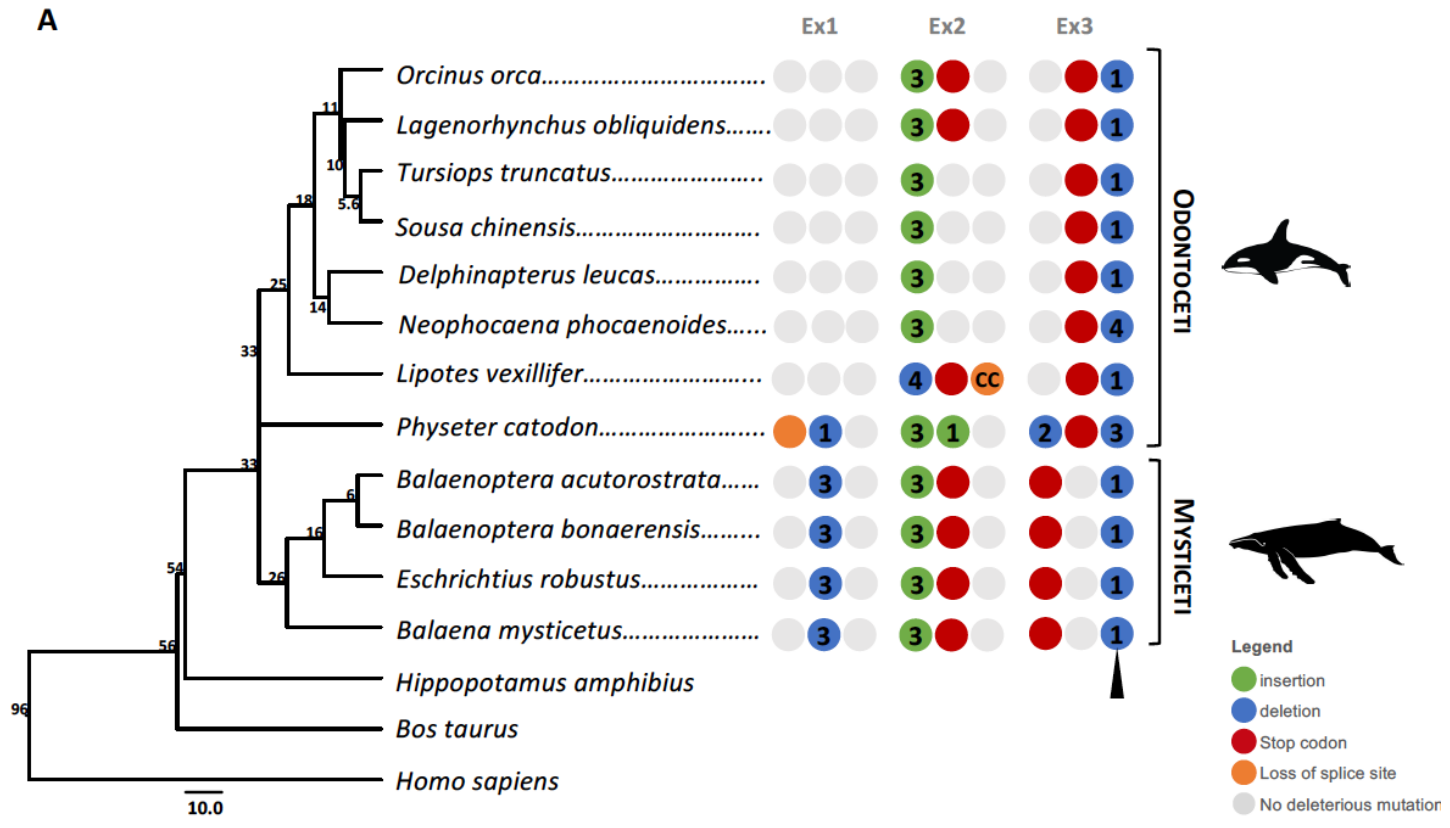

B

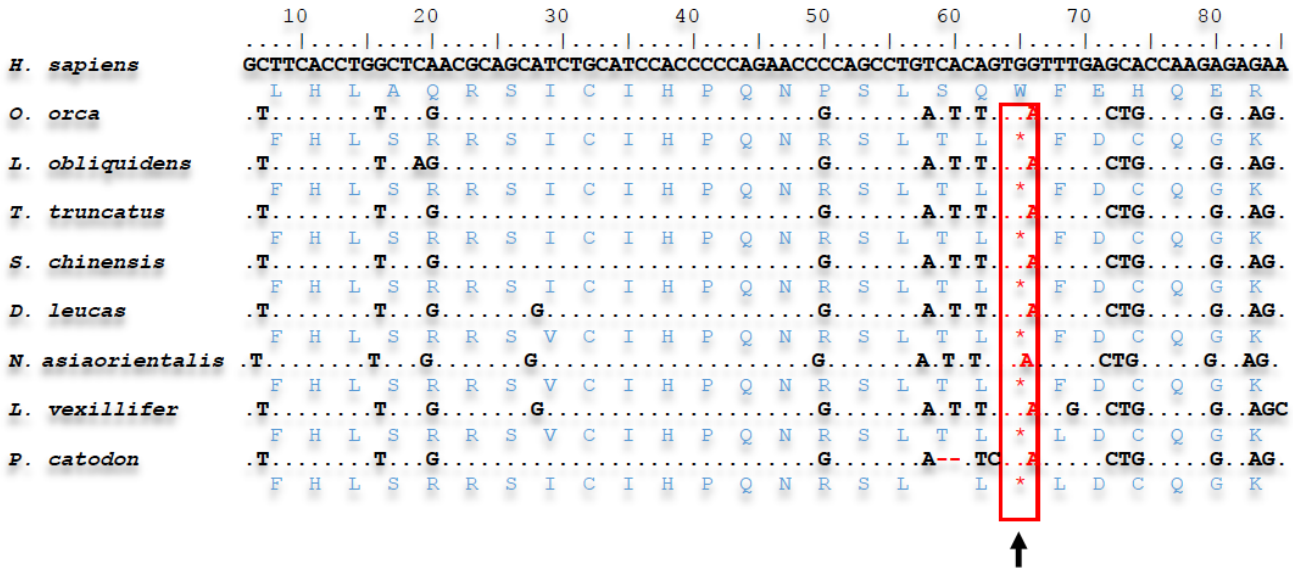

C
H. sapiens
B. acutorostrata
B. bonaerensis
E. robustus
B. mysticetus

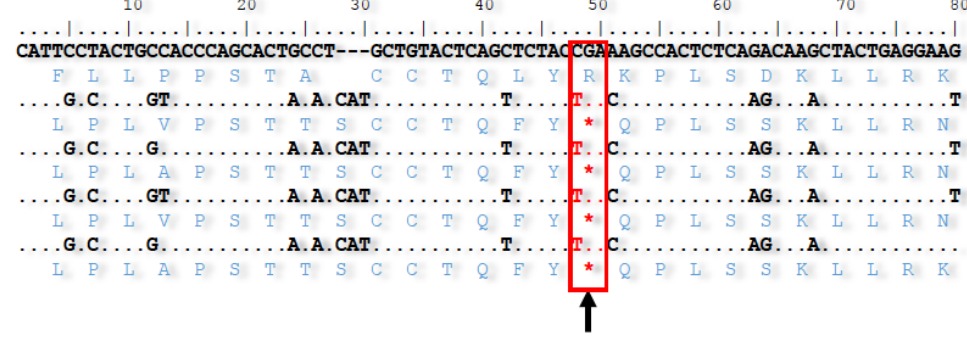

Figure 2: A- Schematic representation of the Ccl27 gene and identified mutations in

497 Cetacea, each group of 3 circles represents one exon, red represents stop codon; orange, 498 non AG-GT splice site; blue deletion and green nucleotide insertion; numbers at tree 499 nodes indicate million years. Number in the circles indicate number of nucleotides 500 inserted or deleted and dark grey circles represent regions or exon not found. B- Sequence 501 alignment of the identified premature stop codon in exon 3 of Odontoceti. C-Sequence alignment of the identified premature stop codon in exon 2 of Mysticeti. 

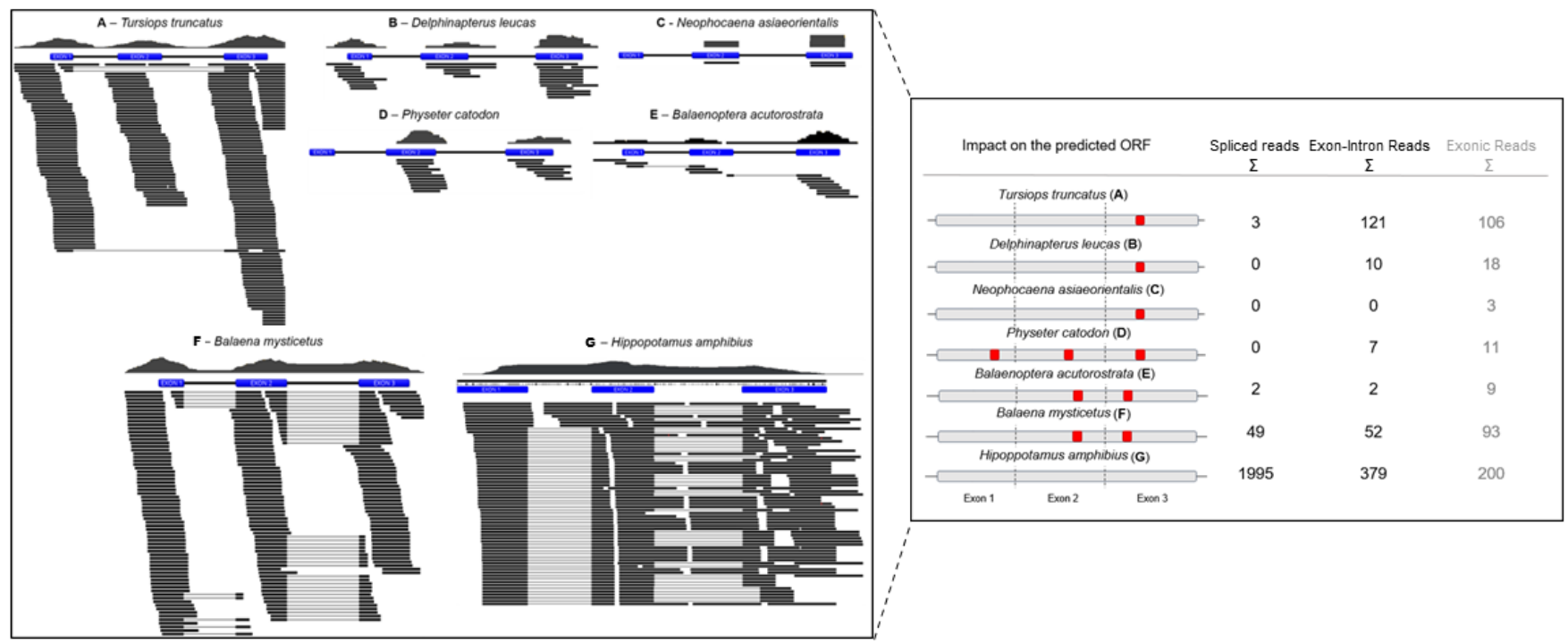

504

505 Figure 3: Gene expression of $C c l 27$ across Cetacea species. In the left box: mapping of

506 the NCBI Sequence Read Archive (SRA) recovered multi-tissue RNA-Seq reads (black)

507 for each of the 7 represented species against the corresponding Ccl27 annotated gene

508 (blue). Right box: impact of the annotated mutations in the open reading frame (ORF) of

509 the $C c l 27$ gene. Premature stop codons are represented with a red squared marker at the

510 corresponding exon. Overall count of RNA-Seq mapped reads for each specie. Reads are

511 classified into spliced reads (reads spanning over two different exons), exon-intron reads

512 (reads containing exonic and intronic sequence) and exonic reads (reads fully overlapping

513 exonic regions).

514 
A
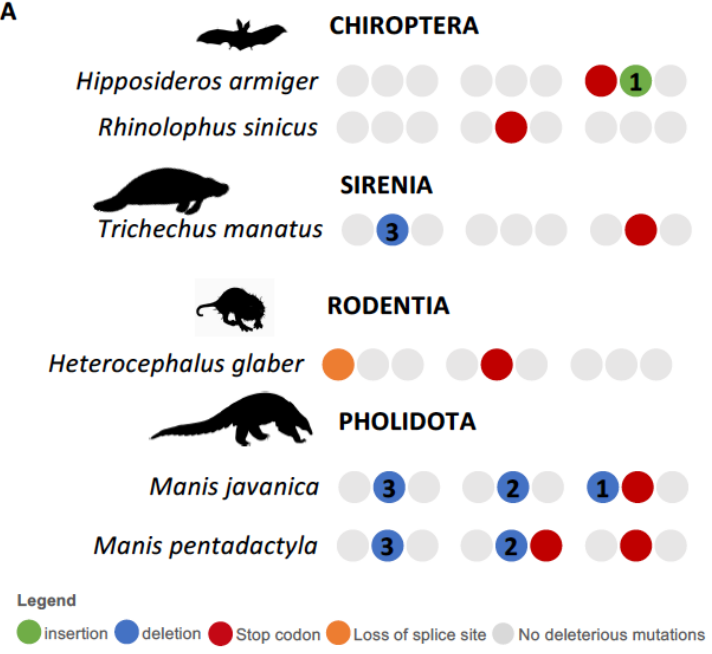

C

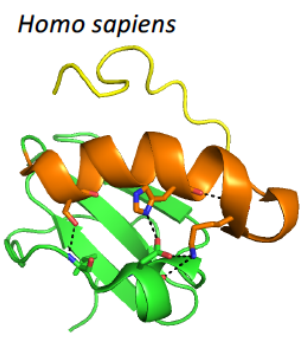

Aotus nancymaae

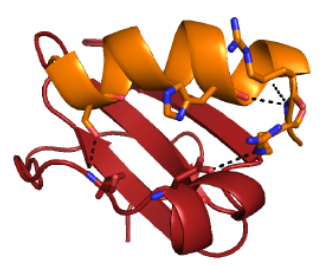

Orcinus orca

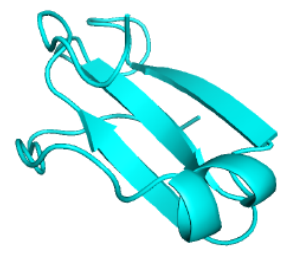

Balaena mysticetus

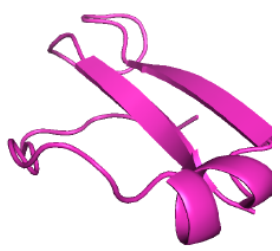

B

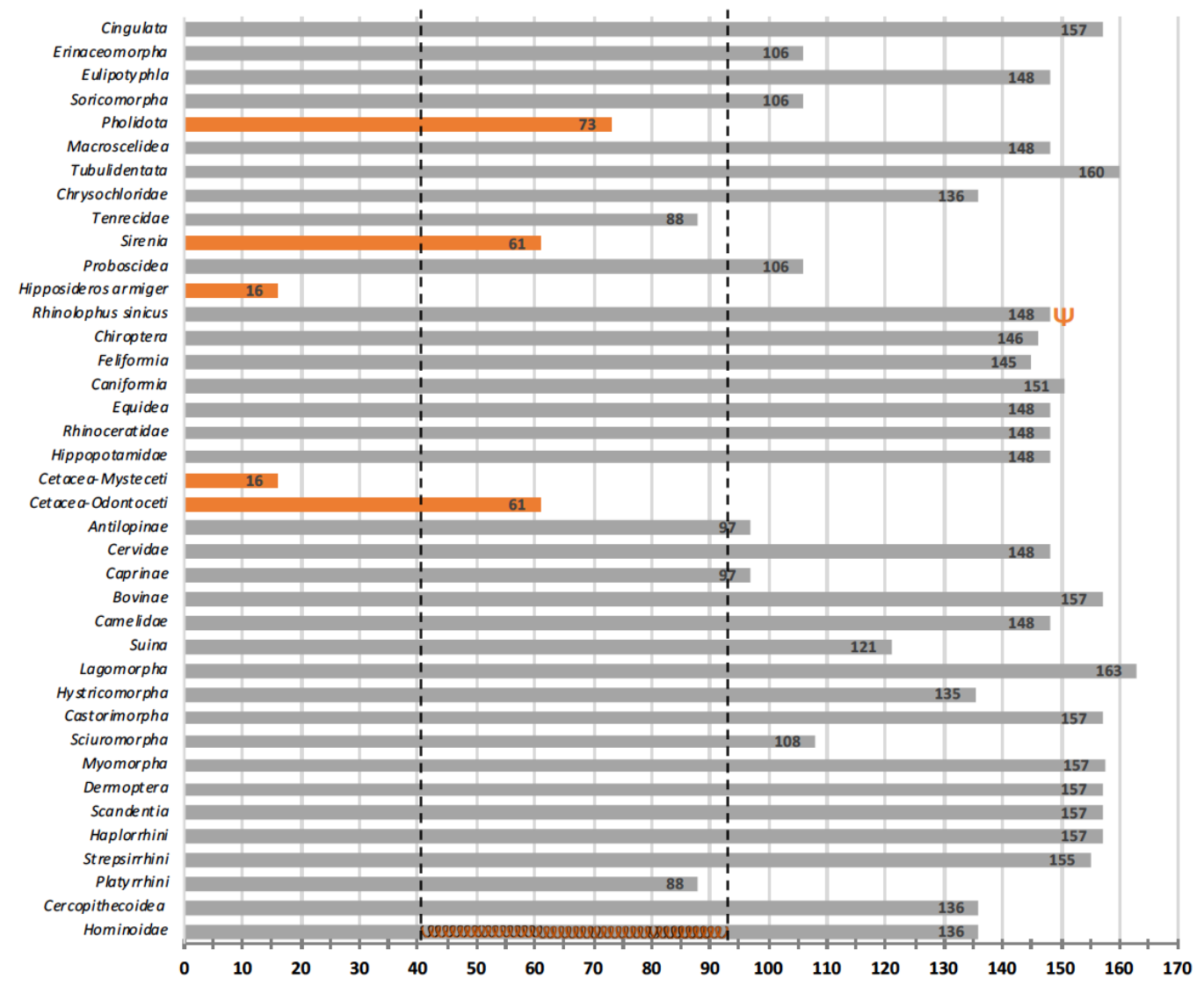

517 Figure 4: A- Gene annotation of $\mathrm{Ccl7}$ in non-cetacean mammals. B- Analysis of exon3

518 length in nucleotides, orange bars highlights species with severe exon 3 truncation, orange

519 helix in Hominoidae bar corresponds to extension C-terminal $\alpha$-helix in human crystal

520 structure (2KUM). C-Comparative analysis of the human crystal structure $2 \mathrm{KUM}$ (green)

521 and calculated homology models in red Aotus nancymaae, blue Orcinus orca and magenta

522 Balaena mysticetus. Structural features highlighted in human in orange terminal $\alpha$-helix,

523 in yellow disordered terminal region. 


\section{Supplementary Information Legends}

525

526 Supplementary Table 1: Accession numbers of the analysed sequences * tagged low-

527 quality, a assembled genomes without annotation.

528 Supplementary Table 2: In-depth description of the available transcriptomic NCBI

529 sequence read archive (SRA) projects, scrutinized in the transcriptomic analysis of the 6

530 represented cetaceans.

531 Supplementary Material 1: SRA validation of the identified mutations in Cetacea

532 Supplementary Material 2: Sequence alignment of $C c l 27$ exon 3 from Cetacea, $H$.

533 amphibius and $H$. sapiens.

534 Supplementary Material 3: SRA validation of inactivating mutations of $C c l 27$

535 transcripts in Cetacea.

536 Supplementary Material 4: SRA Validation of identified mutations in other mammals.

537 Supplementary Material 5: Distribution and phylogenetic analysis of coding Ccl27 in

538 mammals. 